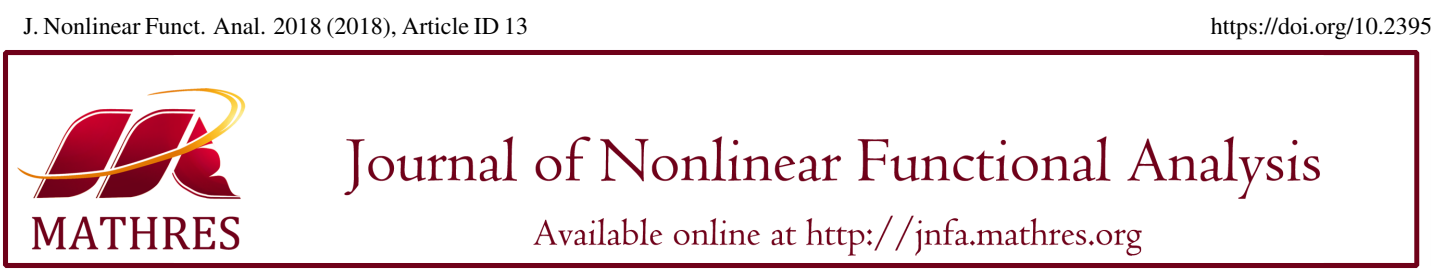

https://doi.org/10.23952/jnfa.2018.13

\title{
PERIODIC BOUNDARY VALUE PROBLEMS WITH DELTA RIEMANN-LIOUVILLE FRACTIONAL DERIVATIVE ON TIME SCALES
}

\author{
İSMAİL YASLAN*, ONUR LİCELİ \\ Department of Mathematics, Pamukkale University, 20070 Denizli, Turkey
}

\begin{abstract}
In this paper, we investigate the existence and the uniqueness of solutions of a periodic boundary value problem for a class of fractional differential equations on time scales. Some fixed point theorems are applied to obtain some new existence results. An illustrative example is also provided.
\end{abstract}

Keywords. Boundary value problem; Fixed point theorem; Fractional differential equation; Positive solution; Time scales.

2010 Mathematics Subject Classification. 34B18, 34A08, 34N05.

\section{INTRODUCTION}

Fractional differential equations appear naturally in various fields of science and engineering, and constitute an important research field. It should be noted that this kind of equations can provide an excellent tool for the description of memory and hereditary properties of various materials and processes. With this advantage, the fractional-order models become more realistic and practical than the classical integerorder models, in which such effects are not taken into account. Recently, the study of the boundary value problems for fractional differential equations has received considerable attention; see $[1,2,3,4,5,6]$ and references therein.

A pioneering work in discrete fractional calculus has been done by Miller and Ross [7]. Especially, Atici and Eloe $[8,9,10]$ contributed to the improvement of the discrete fractional calculus. The existence problems of discrete fractional difference equations have been investigated by many authors; see [9, 10, $11,12,13,14]$ and references therein. And, fractional calculus on time scales was used to unify the fractional differential equations with both continuous and discrete forms; see [15, 16, 17, 18, 19] and the references therein. Some basic definitions and theorems on time scales can be found in the books $[20,21]$. Recently, existence problems of boundary value problems of fractional differential equations on time scales have been studied recently; see [22, 23, 24].

In this paper, we consider the following nonlinear periodic boundary value problem with delta RiemannLiouville fractional derivative on time scales of order $\alpha-1$ :

${ }^{*}$ Corresponding author.

E-mail address: iyaslan@pau.edu.tr (İ. Yaslan).

Received June 20, 2017; Accepted March 6, 2018. 


$$
\left\{\begin{array}{c}
\Delta_{a^{*}}^{\alpha-1} x(t)=f(t, x(t)), t \in J:=[a, b] \cap \mathbb{T}, 2<\alpha<3 \\
x(a)=x(b)=0, x^{\Delta}(a)=x^{\Delta}(b),
\end{array}\right.
$$

where $\mathbb{T}$ is any time scale, $f \in C([a, b] \times \mathbb{R}, \mathbb{R})$ and $\Delta_{a^{*}}^{\alpha-1}$ denotes the delta fractional derivative on time scale $\mathbb{T}$ of order $\alpha-1$ which will be defined later.

The organization of this the paper is as follows. In Section 2, we will give some definitions of delta Riemann-Liouville type fractional integral and delta fractional derivative on time scales and auxiliary lemmas which are needed later. In Section 3, by using the contraction mapping theorem (Banach fixed point theorem) we show that there is a unique solution of BVP (1.1) if $f(t, x)$ satisfies a Lipschitz condition. In the last section, by using some known fixed point theorems, we obtained sufficient conditions for the existence of solutions without the implication of the uniqueness of solutions.

\section{PRELIMINARIES}

To state the main results of this paper, we will need some basic definitions and lemmas.

Let us consider the rd-continuous functions $h_{\alpha}: \mathbb{T} \times \mathbb{T} \rightarrow \mathbb{R}, \alpha \geq 0$ such that

$$
h_{\alpha+1}(t, s)=\int_{s}^{t} h_{\alpha}(\tau, s) \Delta \tau, h_{0}(t, s)=1, \forall s, t \in \mathbb{T},
$$

where $\mathbb{T}$ is a time scale such that $\mathbb{T}^{k}=\mathbb{T}$. Also, we suppose

$$
\int_{\sigma(u)}^{t} h_{\alpha-1}(t, \sigma(\tau)) h_{\beta-1}(\tau, \sigma(u)) \Delta \tau=h_{\alpha+\beta-1}(t, \sigma(u)), \alpha, \beta>1, u<t, u, t \in \mathbb{T},
$$

where $\sigma$ is the forward jump operator.

If $\mathbb{T}=\mathbb{R}$, then $\sigma(t)=t$ and $h_{k}(t, s)=\frac{(t-s)^{k}}{k !}, \forall k \in \mathbb{N}_{0}=\mathbb{N} \cup\{0\}$. We define

$$
h_{\alpha}(t, s)=\frac{(t-s)^{\alpha}}{\Gamma(\alpha+1)}, \alpha>0
$$

which satisfies the properties in (2.1) and (2.2) (see [19]).

If $\mathbb{T}=\mathbb{Z}$, then $\sigma(t)=t+1$ and $h_{k}(t, s)=\frac{(t-s)^{(k)}}{k !}, \quad \forall k \in \mathbb{N}_{0}$, where $t^{(0)}=1, t^{(k)}=\prod_{i=0}^{k-1}(t-i)$. We define

$$
h_{\alpha}(t, s)=\frac{(t-s)^{(\alpha)}}{\Gamma(\alpha+1)}, \alpha>0
$$

where $t^{(\alpha)}=\frac{\Gamma(t+1)}{\Gamma(t-\alpha+1)}$ which satisfies the properties in (2.1) and (2.2) (see [19]).

Definition 2.1. [19] For $\alpha \geq 1$, a time scale delta Riemann-Liouville type fractional integral is defined by

$$
K_{a}^{\alpha} f(t)=\int_{a}^{t} h_{\alpha-1}(t, \sigma(\tau)) f(\tau) \Delta \tau, K_{a}^{0} f=f
$$

where $f \in L_{1}([a, b] \cap \mathbb{T})$ and $t \in[a, b] \cap \mathbb{T}$.

If $\alpha=1$, then we have $K_{a}^{1} f(t)=\int_{a}^{t} f(\tau) \Delta \tau$. 
Definition 2.2. [19] For $\alpha \geq 2, m-1<\alpha \leq m \in \mathbb{N}$, i.e., $m=\ulcorner\alpha\urcorner$ (ceiling of the number) and $v=m-\alpha$, the $\Delta$ - fractional derivative on time scale $\mathbb{T}$ of order $\alpha-1$ is defined by

$$
\Delta_{a *}^{\alpha-1} f(t)=\left(K_{a}^{v+1} f^{\Delta^{m}}\right)(t)=\int_{a}^{t} h_{v}(t, \sigma(\tau)) f^{\Delta^{m}}(\tau) \Delta \tau, \forall t \in[a, b] \cap \mathbb{T},
$$

where $f \in C_{r d}^{m}([a, b] \cap \mathbb{T})$ and $f^{\Delta^{m}}$ is a Lebesgue $\Delta$ - integrable function.

If we take $\alpha=m$, then we have $\Delta_{a *}^{\alpha-1} f(t)=\left(K_{a}^{1} f^{\Delta^{m}}\right)(t)=f^{\Delta^{m-1}}$.

Lemma 2.3. [19] Let $\alpha>2, m-1<\alpha<m \in \mathbb{N}, v=m-\alpha, f \in C_{r d}^{m}(\mathbb{T}), a, b \in \mathbb{T}$, $\mathbb{T}^{k}=\mathbb{T}$. Suppose that $h_{\alpha-2}(s, \sigma(t)), h_{v}(s, \sigma(t))$ is continuous on $([a, b] \cap \mathbb{T})^{2}$. Then, we have

$$
K_{a}^{\alpha-1} \Delta_{a *}^{\alpha-1} f(t)=f(t)+E\left(f^{\Delta^{m}}, \alpha-1, v+1, \mathbb{T}, t\right)-\sum_{k=0}^{m-1} h_{k}(t, a) f^{\Delta^{k}}(a)
$$

where $E\left(f^{\Delta^{m}}, \alpha-1, v+1, \mathbb{T}, t\right)=\int_{a}^{t} f^{\Delta^{m}}(u) \mu(u) h_{\alpha-2}(t, \sigma(u)) h_{v}(u, \sigma(u)) \Delta u$ and $\mu(t)=\sigma(t)-t$.

Lemma 2.4. Assume that $2<\alpha<3, \beta=3-\alpha, x \in C_{r d}^{3}(\mathbb{T}), g \in C_{r d}([a, b] \cap \mathbb{T}), a, b \in \mathbb{T}, a<b, \mathbb{T}^{k}=\mathbb{T}$, and $h_{\alpha-1}(t, \sigma(s))$ is continuous on $J \times J$. Then, a function $x \in C_{r d}^{3}(\mathbb{T})$ is a solution of the boundary value problem

$$
\left\{\begin{array}{c}
\Delta_{a^{*}}^{\alpha-1} x(t)=g(t), t \in[a, b] \cap \mathbb{T}, 2<\alpha<3, \\
x(a)=x(b)=0, x^{\Delta}(a)=x^{\Delta}(b),
\end{array}\right.
$$

if and only if $x$ is a solution of the following integral equation

$$
\begin{aligned}
x(t)= & \int_{a}^{t} h_{\alpha-2}(t, \sigma(\tau))\left(g(\tau)-x^{\Delta^{3}}(\tau) \mu(\tau) h_{\beta}(\tau, \sigma(\tau))\right) \Delta \tau \\
+ & \int_{a}^{b}\left[\left(\frac{t-a}{(b-a)^{2}} h_{2}(b, a)-\frac{h_{2}(t, a)}{b-a}\right) h_{\alpha-2}^{\Delta}(b, \sigma(\tau))-\frac{t-a}{b-a} h_{\alpha-2}(b, \sigma(\tau))\right] \\
& \left(g(\tau)-x^{\Delta^{3}}(\tau) \mu(\tau) h_{\beta}(\tau, \sigma(\tau))\right) \Delta \tau .
\end{aligned}
$$

Proof. Let $x$ be a solution of BVP (2.3). By Lemma 2.3, we have

$$
\begin{aligned}
K_{a}^{\alpha-1} g(t) & =K_{a}^{\alpha-1} \Delta_{a^{*}}^{\alpha-1} x(t) \\
& =x(t)+\int_{a}^{t} x^{\Delta^{3}}(\tau) \mu(\tau) h_{\alpha-2}(t, \sigma(\tau)) h_{\beta}(\tau, \sigma(\tau)) \Delta \tau-\sum_{k=0}^{2} h_{k}(t, a) x^{\Delta^{k}}(a) .
\end{aligned}
$$

Then, we obtain

$$
\begin{aligned}
x(t) & =\int_{a}^{t} h_{\alpha-2}(t, \sigma(\tau)) g(\tau) \Delta \tau-\int_{a}^{t} h_{\alpha-2}(t, \sigma(\tau)) x^{\Delta^{3}}(\tau) \mu(\tau) h_{\beta}(\tau, \sigma(\tau)) \Delta \tau+x(a) \\
& +h_{1}(t, a) x^{\Delta}(a)+h_{2}(t, a) x^{\Delta^{2}}(a) .
\end{aligned}
$$


Using the differentiation formula [21, Theorem 1.117], we have

$$
x^{\Delta}(t)=\int_{a}^{t} h_{\alpha-2}^{\Delta}(t, \sigma(\tau))\left(g(\tau)-x^{\Delta^{3}}(\tau) \mu(\tau) h_{\beta}(\tau, \sigma(\tau))\right) \Delta \tau+x^{\Delta}(a)+(t-a) x^{\Delta^{2}}(a) .
$$

By using the second boundary condition, we get

$$
x^{\Delta^{2}}(a)=-\frac{1}{b-a} \int_{a}^{b} h_{\alpha-2}^{\Delta}(b, \sigma(\tau))\left(g(\tau)-x^{\Delta^{3}}(\tau) \mu(\tau) h_{\beta}(\tau, \sigma(\tau))\right) \Delta \tau .
$$

From the first boundary condition, we find

$$
\begin{aligned}
x^{\Delta}(a)= & -\frac{1}{b-a} \int_{a}^{b}\left[h_{\alpha-2}(b, \sigma(\tau))-\frac{h_{2}(b, a) h_{\alpha-2}^{\Delta}(b, \sigma(\tau))}{b-a}\right] \\
& \left(g(\tau)-x^{\Delta^{3}}(\tau) \mu(\tau) h_{\beta}(\tau, \sigma(\tau))\right) \Delta \tau .
\end{aligned}
$$

Hence, it follows from (2.5) that (2.4). The converse of the lemma follows by a direct computation. This completes the proof.

$C_{r d}^{3}(\mathbb{T})$ is a Banach space with the norm $\|x\|=\max _{t \in J}|x(t)|+\max _{t \in J}\left|x^{\Delta^{3}}(t)\right|$ (see [25]). The solutions of BVP (1.1) are the fixed points of the operator $A: C_{r d}^{3}(\mathbb{T}) \rightarrow C_{r d}^{3}(\mathbb{T})$ defined by

$$
\begin{aligned}
A x(t) & =\int_{a}^{t} h_{\alpha-2}(t, \sigma(\tau))\left(f(\tau, x(\tau))-x^{\Delta^{3}}(\tau) \mu(\tau) h_{\beta}(\tau, \sigma(\tau))\right) \Delta \tau \\
& +\int_{a}^{b} k(t, \tau)\left(f(\tau, x(\tau))-x^{\Delta^{3}}(\tau) \mu(\tau) h_{\beta}(\tau, \sigma(\tau))\right) \Delta \tau,
\end{aligned}
$$

where $k(t, \tau)=\left(\frac{t-a}{(b-a)^{2}} h_{2}(b, a)-\frac{h_{2}(t, a)}{b-a}\right) h_{\alpha-2}^{\Delta}(b, \sigma(\tau))-\frac{t-a}{b-a} h_{\alpha-2}(b, \sigma(\tau))$.

For the sake of convenience, we set

$$
M=\max _{t \in J}\left(\int_{a}^{t}\left|h_{\alpha-2}(t, \sigma(\tau))\right| \Delta \tau+\int_{a}^{b}|k(t, \tau)| \Delta \tau\right)+\max _{t \in J} \int_{a}^{t}\left|h_{\alpha-2}^{\Delta^{3}}(t, \sigma(\tau))\right| \Delta \tau
$$

and

$$
\begin{aligned}
N & =\max _{t \in J}\left(\int_{a}^{t}\left|h_{\alpha-2}(t, \sigma(\tau)) \mu(\tau) h_{\beta}(\tau, \sigma(\tau))\right| \Delta \tau+\int_{a}^{b}\left|k(t, \tau) \mu(\tau) h_{\beta}(\tau, \sigma(\tau))\right| \Delta \tau\right) \\
& +\max _{t \in J} \int_{a}^{t}\left|h_{\alpha-2}^{\Delta^{3}}(t, \sigma(\tau)) \mu(\tau) h_{\beta}(\tau, \sigma(\tau))\right| \Delta \tau .
\end{aligned}
$$

Lemma 2.5. Assume the following two conditions hold:

$(H 1)|f(t, x)| \leq \phi(t) \psi(|x|)$ for all $t \in J, x \in C_{r d}^{3}(\mathbb{T})$, where $\phi: J \rightarrow[0, \infty)$ and $\psi:[0, \infty) \rightarrow[0, \infty)$ are continuous and nondecreasing.

(H2) The functions $h_{\alpha-2}(t, \sigma(\tau)), h_{\alpha-2}^{\Delta^{3}}(t, \sigma(\tau)), h_{2}(t, a)$ and $\mu(t) h_{\beta}(t, \sigma(t))$ are continuous for $t \in J$ and $\tau \in J$.

Then, $A: C_{r d}^{3}(\mathbb{T}) \rightarrow C_{r d}^{3}(\mathbb{T})$ is completely continuous. 
Proof. We divide the proof into two steps.

Step 1: We show that $A$ is continuous. Let $x_{n}$ be a sequence such that $x_{n} \rightarrow x \in C_{r d}^{3}(\mathbb{T})$. Then, we obtain that

$$
\begin{aligned}
\left|\left(A x_{n}\right)(t)-(A x)(t)\right| & \leq \int_{a}^{t}\left|h_{\alpha-2}(t, \sigma(\tau))\right|\left|f\left(\tau, x_{n}(\tau)\right)-f(\tau, x(\tau))\right| \Delta \tau \\
& +\int_{a}^{t}\left|h_{\alpha-2}(t, \sigma(\tau)) \mu(\tau) h_{\beta}(\tau, \sigma(\tau))\right|\left|x_{n}^{\Delta^{3}}(\tau)-x^{\Delta^{3}}(\tau)\right| \Delta \tau \\
& +\int_{a}^{b}|k(t, \tau)|\left|f\left(\tau, x_{n}(\tau)\right)-f(\tau, x(\tau))\right| \Delta \tau \\
& +\int_{a}^{b}\left|k(t, \tau) \mu(\tau) h_{\beta}(\tau, \sigma(\tau))\right|\left|x_{n}^{\Delta^{3}}(\tau)-x^{\Delta^{3}}(\tau)\right| \Delta \tau
\end{aligned}
$$

and

$$
\begin{aligned}
\left|\left(A x_{n}\right)^{\Delta^{3}}(t)-(A x)^{\Delta^{3}}(t)\right| & \leq \int_{a}^{t}\left|h_{\alpha-2}^{\Delta^{3}}(t, \sigma(\tau))\right|\left|f\left(\tau, x_{n}(\tau)\right)-f(\tau, x(\tau))\right| \Delta \tau \\
& +\int_{a}^{t}\left|h_{\alpha-2}^{\Delta^{3}}(t, \sigma(\tau)) \mu(\tau) h_{\beta}(\tau, \sigma(\tau))\right|\left|x_{n}^{\Delta^{3}}(\tau)-x^{\Delta^{3}}(\tau)\right| \Delta \tau .
\end{aligned}
$$

From $f \in C([a, b] \times \mathbb{R}, \mathbb{R}),(H 2)$ and $\left\|x_{n}-x\right\| \rightarrow 0$ as $n \rightarrow \infty$, it follows that $\left\|A x_{n}-A x\right\| \rightarrow 0$ as $n \rightarrow \infty$. So, $A$ is continuous.

Step 2: We show that the image of any bounded subset $\Omega$ of $C_{r d}^{3}(\mathbb{T})$ under $A$ is relatively compact in $C_{r d}^{3}(\mathbb{T})$. For each $x \in \Omega=\left\{x \in C_{r d}^{3}(\mathbb{T}):\|x\| \leq r\right\}$, we obtain

$$
\begin{aligned}
|(A x)(t)| & \leq \int_{a}^{t}\left|h_{\alpha-2}(t, \sigma(\tau))\right||f(\tau, x(\tau))| \Delta \tau \\
& +\int_{a}^{t}\left|h_{\alpha-2}(t, \sigma(\tau)) \mu(\tau) h_{\beta}(\tau, \sigma(\tau))\right|\left|x^{\Delta^{3}}(\tau)\right| \Delta \tau \\
& +\int_{a}^{b}|k(t, \tau)||f(\tau, x(\tau))| \Delta \tau \\
& +\int_{a}^{b}\left|k(t, \tau) \mu(\tau) h_{\beta}(\tau, \sigma(\tau))\right|\left|x^{\Delta^{3}}(\tau)\right| \Delta \tau \\
& \leq \phi(b) \psi(r) \int_{a}^{t}\left|h_{\alpha-2}(t, \sigma(\tau))\right| \Delta \tau+\|x\| \int_{a}^{t}\left|h_{\alpha-2}(t, \sigma(\tau)) \mu(\tau) h_{\beta}(\tau, \sigma(\tau))\right| \Delta \tau \\
& +\phi(b) \psi(r) \int_{a}^{b}|k(t, \tau)| \Delta \tau+\|x\| \int_{a}^{b}\left|k(t, \tau) \mu(\tau) h_{\beta}(\tau, \sigma(\tau))\right| \Delta \tau
\end{aligned}
$$


and

$$
\begin{aligned}
\left|(A x)^{\Delta^{3}}(t)\right| & \leq \int_{a}^{t}\left|h_{\alpha-2}^{\Delta^{3}}(t, \sigma(\tau))\right||f(\tau, x(\tau))| \Delta \tau \\
& \left.+\int_{a}^{t}\left|h_{\alpha-2}^{\Delta^{3}}(t, \sigma(\tau)) \mu(\tau) h_{\beta}(\tau, \sigma(\tau))\right| \mid x^{\Delta^{3}}(\tau)\right) \mid \Delta \tau \\
& \leq \phi(b) \psi(r) \int_{a}^{t}\left|h_{\alpha-2}^{\Delta^{3}}(t, \sigma(\tau))\right| \Delta \tau \\
& +\|x\| \int_{a}^{t}\left|h_{\alpha-2}^{\Delta^{3}}(t, \sigma(\tau)) \mu(\tau) h_{\beta}(\tau, \sigma(\tau))\right| \Delta \tau
\end{aligned}
$$

Therefore,

$$
\begin{aligned}
\|A x\| & \leq \phi(b) \psi(r) M+\|x\| N \\
& \leq \phi(b) \psi(r) M+r N,
\end{aligned}
$$

that is, $A \Omega$ is a bounded set.

Now we show that $A \Omega$ is equicontinuous on $J$. For each $t_{1}, t_{2} \in J$, without loss of generality, we may assume that $t_{1}<t_{2}$. For all $x \in \Omega$, one can see that

$$
\begin{aligned}
& \left|A x\left(t_{2}\right)-A x\left(t_{1}\right)\right| \\
\leq & \mid \int_{a}^{t_{2}} h_{\alpha-2}\left(t_{2}, \sigma(\tau)\right)\left(f(\tau, x(\tau))-x^{\Delta^{3}}(\tau) \mu(\tau) h_{\beta}(\tau, \sigma(\tau))\right) \Delta \tau \\
- & \int_{a}^{t_{1}} h_{\alpha-2}\left(t_{1}, \sigma(\tau)\right)\left(f(\tau, x(\tau))-x^{\Delta^{3}}(\tau) \mu(\tau) h_{\beta}(\tau, \sigma(\tau))\right) \Delta \tau \mid \\
+ & \mid \int_{a}^{b} k\left(t_{2}, \tau\right)\left(f(\tau, x(\tau))-x^{\Delta^{3}}(\tau) \mu(\tau) h_{\beta}(\tau, \sigma(\tau))\right) \Delta \tau \\
- & \int_{a}^{b} k\left(t_{1}, \tau\right)\left(f(\tau, x(\tau))-x^{\Delta^{3}}(\tau) \mu(\tau) h_{\beta}(\tau, \sigma(\tau))\right) \Delta \tau \mid \\
\leq & \int_{a}^{t_{1}}\left|h_{\alpha-2}\left(t_{2}, \sigma(\tau)\right)-h_{\alpha-2}\left(t_{1}, \sigma(\tau)\right)\right|\left|f(\tau, x(\tau))-x^{\Delta^{3}}(\tau) \mu(\tau) h_{\beta}(\tau, \sigma(\tau))\right| \Delta \tau \\
+ & \int_{t_{1}}^{t_{2}}\left|h_{\alpha-2}\left(t_{2}, \sigma(\tau)\right)\right|\left|f(\tau, x(\tau))-x^{\Delta^{3}}(\tau) \mu(\tau) h_{\beta}(\tau, \sigma(\tau))\right| \Delta \tau \\
+ & \int_{a}^{b}\left|k\left(t_{2}, \tau\right)-k\left(t_{1}, \tau\right)\right|\left|f(\tau, x(\tau))-x^{\Delta^{3}}(\tau) \mu(\tau) h_{\beta}(\tau, \sigma(\tau))\right| \Delta \tau
\end{aligned}
$$


and

$$
\begin{aligned}
\left|(A x)^{\Delta^{3}}\left(t_{2}\right)-(A x)^{\Delta^{3}}\left(t_{1}\right)\right| & \leq \mid \int_{a}^{t_{2}} h_{\alpha-2}^{\Delta^{3}}\left(t_{2}, \sigma(\tau)\right)\left(f(\tau, x(\tau))-x^{\Delta^{3}}(\tau) \mu(\tau) h_{\beta}(\tau, \sigma(\tau))\right) \Delta \tau \\
& -\int_{a}^{t_{1}} h_{\alpha-2}^{\Delta^{3}}\left(t_{1}, \sigma(\tau)\right)\left(f(\tau, x(\tau))-x^{\Delta^{3}}(\tau) \mu(\tau) h_{\beta}(\tau, \sigma(\tau))\right) \Delta \tau \mid \\
& \leq \int_{a}^{t_{1}}\left|h_{\alpha-2}^{\Delta^{3}}\left(t_{2}, \sigma(\tau)\right)-h_{\alpha-2}^{\Delta^{3}}\left(t_{1}, \sigma(\tau)\right)\right| \\
& +\int_{t_{1}}^{t_{2}}\left|h_{\alpha-2}^{\Delta^{3}}\left(t_{2}, \sigma(\tau)\right)\right|\left|f(\tau, x(\tau))-x^{\Delta^{3}}(\tau) \mu(\tau) h_{\beta}(\tau, \sigma(\tau))\right| \Delta \tau .
\end{aligned}
$$

Since the functions $f, h_{\alpha-2}(t, \sigma(\tau)), h_{\alpha-2}^{\Delta^{3}}(t, \sigma(\tau)), h_{2}(t, a)$ and $\mu(t) h_{\beta}(t, \sigma(t))$ are continuous from $(H 2)$, we have $\left|A x\left(t_{2}\right)-A x\left(t_{1}\right)\right| \rightarrow 0$ and $\left|(A x)^{\Delta^{3}}\left(t_{2}\right)-(A x)^{\Delta^{3}}\left(t_{1}\right)\right| \rightarrow 0$ for $t_{1} \rightarrow t_{2}$. It yields that $A \Omega$ is equicontinuous in $C_{r d}^{3}(\mathbb{T})$.

As a consequence of those steps, we obtain that $A$ is completely continuous operator. This completes the proof.

\section{The LipsChitz CASE}

In this section, we will use the well-known contraction mapping theorem named also as the Banach fixed point theorem.

Theorem 3.1. Assume that (H2) holds. In addition, we suppose that (H3) Let the function $f(t, x)$ satisfy the following Lipschitz condition: there is a constant $L>0$ such that

$$
|f(t, x)-f(t, y)| \leq L|x-y|, \text { for each } t \in J,
$$

for all $x$ and $y$ in $C_{r d}^{3}(\mathbb{T})$. In addition, $L M+N<1$, where $M$ and $N$ are defined in (2.7) and (2.8), respectively. Then, $B V P(1.1)$ has a unique solution in $C_{r d}^{3}(\mathbb{T})$.

Proof. For $x, y \in C_{r d}^{3}(\mathbb{T})$ and $t \in J$, by using (3.1) we have

$$
\begin{aligned}
|(A x)(t)-(A y)(t)| & \leq \int_{a}^{t}\left|h_{\alpha-2}(t, \sigma(\tau))(f(\tau, x(\tau))-f(\tau, y(\tau)))\right| \Delta \tau \\
& +\int_{a}^{t}\left|h_{\alpha-2}(t, \sigma(\tau)) \mu(\tau) h_{\beta}(\tau, \sigma(\tau))\left(x^{\Delta^{3}}(\tau)-y^{\Delta^{3}}(\tau)\right)\right| \Delta \tau \\
& +\int_{a}^{b}|k(t, \tau)(f(\tau, x(\tau))-f(\tau, y(\tau)))| \Delta \tau \\
& +\int_{a}^{b}\left|k(t, \tau) \mu(\tau) h_{\beta}(\tau, \sigma(\tau))\left(x^{\Delta^{3}}(\tau)-y^{\Delta^{3}}(\tau)\right)\right| \Delta \tau
\end{aligned}
$$




$$
\begin{aligned}
& \leq L \int_{a}^{t}\left|h_{\alpha-2}(t, \sigma(\tau))\right||x(\tau)-y(\tau)| \Delta \tau \\
& +\int_{a}^{t}\left|h_{\alpha-2}(t, \sigma(\tau)) \mu(\tau) h_{\beta}(\tau, \sigma(\tau))\right|\left|x^{\Delta^{3}}(\tau)-y^{\Delta^{3}}(\tau)\right| \Delta \tau \\
& +L \int_{a}^{b}|k(t, \tau)||x(\tau)-y(\tau)| \Delta \tau \\
& +\int_{a}^{b}\left|k(t, \tau) \mu(\tau) h_{\beta}(\tau, \sigma(\tau))\right|\left|\left(x^{\Delta^{3}}(\tau)-y^{\Delta^{3}}(\tau)\right)\right| \Delta \tau \\
& \leq \quad L\|x-y\|\left(\int_{a}^{t}\left|h_{\alpha-2}(t, \sigma(\tau))\right| \Delta \tau+\int_{a}^{b}|k(t, \tau)| \Delta \tau\right) \\
& +\quad\|x-y\|\left(\int_{a}^{t}\left|h_{\alpha-2}(t, \sigma(\tau)) \mu(\tau) h_{\beta}(\tau, \sigma(\tau))\right| \Delta \tau\right. \\
& \left.+\int_{a}^{b}\left|k(t, \tau) \mu(\tau) h_{\beta}(\tau, \sigma(\tau))\right| \Delta \tau\right)
\end{aligned}
$$

and

$$
\begin{aligned}
\left|(A x)^{\Delta^{3}}(t)-(A y)^{\Delta^{3}}(t)\right| & \leq \int_{a}^{t}\left|h_{\alpha-2}^{\Delta^{3}}(t, \sigma(\tau))(f(\tau, x(\tau))-f(\tau, y(\tau)))\right| \Delta \tau \\
& +\int_{a}^{t}\left|h_{\alpha-2}^{\Delta^{3}}(t, \sigma(\tau)) \mu(\tau) h_{\beta}(\tau, \sigma(\tau))\left(x^{\Delta^{3}}(\tau)-y^{\Delta^{3}}(\tau)\right)\right| \Delta \tau \\
& \leq L \int_{a}^{t}\left|h_{\alpha-2}^{\Delta^{3}}(t, \sigma(\tau))\right||x(\tau)-y(\tau)| \Delta \tau \\
& +\int_{a}^{t}\left|h_{\alpha-2}^{\Delta^{3}}(t, \sigma(\tau)) \mu(\tau) h_{\beta}(\tau, \sigma(\tau))\right|\left|x^{\Delta^{3}}(\tau)-y^{\Delta^{3}}(\tau)\right| \Delta \tau \\
& \leq L\|x-y\| \int_{a}^{t}\left|h_{\alpha-2}^{\Delta^{3}}(t, \sigma(\tau))\right| \Delta \tau \\
& +\|x-y\| \int_{a}^{t}\left|h_{\alpha-2}^{\Delta^{3}}(t, \sigma(\tau)) \mu(\tau) h_{\beta}(\tau, \sigma(\tau))\right| \Delta \tau .
\end{aligned}
$$

Then, we obtain

$$
\begin{aligned}
\|A x-A y\| & =\max _{t \in J}|(A x)(t)-(A y)(t)|+\max _{t \in J}\left|(A x)^{\Delta^{3}}(t)-(A y)^{\Delta^{3}}(t)\right| \\
& \leq(L M+N)\|x-y\| \\
& =\lambda\|x-y\|,
\end{aligned}
$$


where $\lambda=L M+N \in(0,1)$. So, $A$ is a contraction mapping and the theorem is proved.

In the next theorem, the function $f(t, x)$ satisfies a Lipschitz condition not on the whole $C_{r d}^{3}(\mathbb{T})$ but on a subset.

Theorem 3.2. Assume that (H2) holds. Also, we suppose that

(H4) There exists a number $r>0$ such that

$$
|f(t, x)-f(t, y)| \leq L|x-y| \text { for each } t \in J
$$

for all $x$ and $y$ in $S=\left\{x \in C_{r d}^{3}(\mathbb{T}):\|x\| \leq r\right\}$, where $L>0$ is a constant which may depend on $r$. In addition, $L M+N<1$, where $M$ and $N$ are defined in (2.7) and (2.8), respectively..

(H5) $\lim _{x \rightarrow 0} \frac{f(t, x)}{x}=0$.

Then, BVP (1.1) has a unique solution $x \in C_{r d}^{3}(\mathbb{T})$ with $\max _{t \in J}|x(t)|+\max _{t \in J}\left|x^{\Delta^{3}}(t)\right| \leq r$.

Proof. From (H5), we find that there exists a constant $r>0$ such that $|f(t, x)| \leq \delta|x|$ for $0<|x| \leq r$, where $\delta>0$ is a constant satisfying $\delta M+N \leq 1$. Let us take $S=\left\{x \in C_{r d}^{3}(\mathbb{T}):\|x\| \leq r\right\}$. Obviously, $S$ is a closed subset of $C_{r d}^{3}(\mathbb{T})$. Let $A: C_{r d}^{3}(\mathbb{T}) \rightarrow C_{r d}^{3}(\mathbb{T})$ be the operator defined in (2.6). For $x$ and $y$ in $S$, taking into account $(H 4)$, in exactly the same way as in the proof of Theorem 3.1 we can get $\|A x-A y\| \leq \lambda\|x-y\|$, where $0<\lambda<1$.

It remains to show that $A$ maps $S$ into itself. If $x \in S$, then we obtain

$$
\begin{aligned}
|(A x)(t)| \leq & \int_{a}^{t}\left|h_{\alpha-2}(t, \sigma(\tau))\right||f(\tau, x(\tau))| \Delta \tau \\
& +\int_{a}^{t}\left|h_{\alpha-2}(t, \sigma(\tau)) \mu(\tau) h_{\beta}(\tau, \sigma(\tau))\right|\left|x^{\Delta^{3}}(\tau)\right| \Delta \tau \\
& +\int_{a}^{b}|k(t, \tau)||f(\tau, x(\tau))| \Delta \tau \\
& +\int_{a}^{b}\left|k(t, \tau) \mu(\tau) h_{\beta}(\tau, \sigma(\tau))\right|\left|x^{\Delta^{3}}(\tau)\right| \Delta \tau \\
& \leq \delta^{b}\|x\|\left(\int_{a}^{t}\left|h_{\alpha-2}(t, \sigma(\tau))\right| \Delta \tau+\int_{a}^{b}|k(t, \tau)| \Delta \tau\right) \\
& +\|x\|\left(\int_{a}^{t}\left|h_{\alpha-2}(t, \sigma(\tau)) \mu(\tau) h_{\beta}(\tau, \sigma(\tau))\right| \Delta \tau\right. \\
& \left.+\int_{a}^{b}\left|k(t, \tau) \mu(\tau) h_{\beta}(\tau, \sigma(\tau))\right| \Delta \tau\right)
\end{aligned}
$$

and 


$$
\begin{aligned}
\left|(A x)^{\Delta^{3}}(t)\right| & \leq \int_{a}^{t}\left|h_{\alpha-2}^{\Delta^{3}}(t, \sigma(\tau))\right||f(\tau, x(\tau))| \Delta \tau \\
& \left.+\int_{a}^{t}\left|h_{\alpha-2}^{\Delta^{3}}(t, \sigma(\tau)) \mu(\tau) h_{\beta}(\tau, \sigma(\tau))\right| \mid x^{\Delta^{3}}(\tau)\right) \mid \Delta \tau \\
& \leq \delta\|x\|\left(\int_{a}^{t}\left|h_{\alpha-2}^{\Delta^{3}}(t, \sigma(\tau))\right| \Delta \tau\right) \\
& +\|x\|\left(\int_{a}^{t}\left|h_{\alpha-2}^{\Delta^{3}}(t, \sigma(\tau)) \mu(\tau) h_{\beta}(\tau, \sigma(\tau))\right| \Delta \tau\right) .
\end{aligned}
$$

Since $\|A x\| \leq\|x\|(\delta M+N) \leq\|x\| \leq r$, we have $A: S \rightarrow S$.

Now the contraction mapping theorem can be applied to obtain a unique solution of (2.4) in $S$, and the proof is complete.

Example 3.3. Let $\mathbb{T}=2 \mathbb{Z}=\{2 k: k \in \mathbb{Z}\}$ and define

$$
h_{\alpha}(t, s)=\frac{2^{\alpha}\left(\frac{t-s}{2}\right)^{(\alpha)}}{\Gamma(\alpha+1)},
$$

where $t^{(\alpha)}=\frac{\Gamma(t+1)}{\Gamma(t-\alpha+1)}$ which satisfies the properties in (2.1) and (2.2). Consider the following boundary value problem

$$
\left\{\begin{aligned}
\Delta_{0^{*}}^{\frac{3}{2}} x(t) & =\frac{t}{40} \frac{|x(t)|}{1+|x(t)|}, t \in J:=[0,20] \cap \mathbb{T}, \\
x(0) & =x(20)=0, x^{\Delta}(0)=x^{\Delta}(20) .
\end{aligned}\right.
$$

Then, we have $M \approx 0.6232, N \approx 0.4532,|f(t, x)-f(t, y)| \leq \frac{1}{2}|x-y|$ and $L M+N \approx 0.7648<1$. Since all the conditions of Theorem 3.1 are satisfied, BVP (3.2) has a unique solution in $C_{r d}^{3}(\mathbb{T})$.

\section{EXistence of Solutions}

Theorem 4.1. [26, 27] Let $E$ be a Banach space. Assume that $A: E \rightarrow E$ is completely continuous operator and the set $V=\{u \in E: u=\lambda A u, 0<\lambda<1\}$ is bounded. Then A has a fixed point in $E$.

Theorem 4.2. If the conditions $(H 1)$ and $(H 2)$ hold, then $B V P(1.1)$ has at least one solution in $C_{r d}^{3}(\mathbb{T})$.

Proof. From Lemma 2.5, $A: C_{r d}^{3}(\mathbb{T}) \rightarrow C_{r d}^{3}(\mathbb{T})$ is completely continuous operator. Now, we will show that the set $V=\left\{x \in C_{r d}^{3}(\mathbb{T}): x=\lambda A x\right.$ for some $\left.0<\lambda<1\right\}$ is bounded. For all $x \in V$, we have

$$
\begin{aligned}
\|x\| & =\|\lambda A x\| \\
& \leq \lambda \phi(b) \psi(r) M+\lambda\|x\| N
\end{aligned}
$$

thanks to (2.9). Then we obtain $\|x\| \leq \frac{\lambda \phi(b) \psi(r) M}{1-\lambda N}$, which yields that the set $\mathrm{V}$ is bounded. As a consequence of Theorem 4.1, BVP (1.1) has at least one solution. This completes the proof. 
Theorem 4.3. [26, 27] Let $E$ be a Banach space. Assume that $\Omega$ is an open bounded subset of $E$ with $0 \in \Omega$ and let $A: \bar{\Omega} \rightarrow E$ be a completely continuous operator such that

$$
\|A u\| \leq\|u\|, \forall x \in \partial \Omega .
$$

Then A has a fixed point in $\bar{\Omega}$.

Theorem 4.4. If conditions (H2) and (H5) hold, then BVP (1.1) has at least one solution.

Proof. Since $\lim _{x \rightarrow 0} \frac{f(t, x)}{x}=0$, there exists a constant $r>0$ such that $|f(t, x)| \leq \delta|x|$ for $0<|x|<r$, where $\delta>0$ is a constant satisfying $\delta M+N<1$. Let us take $\Omega=\left\{x \in C_{r d}^{3}(\mathbb{T}):\|x\|<r\right\}$. Since the function $f$ satisfies condition $(H 1)$ by taking $\phi(t)=\delta$ and $\psi(|x|)=|x|, A: \bar{\Omega} \rightarrow C_{r d}^{3}(\mathbb{T})$ is completely continuous operator from Lemma 2.5. If we take $x \in \partial \Omega$, then we obtain $\|A x\| \leq r$ as in the proof of Theorem 3.2. It follows that $\|A x\| \leq\|x\|, \forall x \in \partial \Omega$. Therefore, by means of Theorem 4.3 the operator A has at least one fixed point in $\bar{\Omega}$. Thus, BVP (1.1) has at least one solution $u \in \bar{\Omega}$.

Corollary 4.5. Suppose that (H1) and (H2) hold. If $\phi(b) M+N \leq 1$ and $\psi(z) \leq z, \forall z \in[0, \infty)$, then $B V P(1.1)$ has at least one solution.

Example 4.6. Let $\mathbb{T}=q \mathbb{Z}=\{q k: k \in \mathbb{Z}\}$ and define

$$
h_{\alpha}(t, s)=\frac{q^{\alpha}\left(\frac{t-s}{q}\right)^{(\alpha)}}{\Gamma(\alpha+1)},
$$

where $t^{(\alpha)}=\frac{\Gamma(t+1)}{\Gamma(t-\alpha+1)}$ which satisfies the properties in (2.1) and (2.2). Consider the following boundary value problem

$$
\left\{\begin{array}{c}
\Delta_{0^{*}}^{\alpha-1} x(t)=\frac{x(1-\cos (t x))}{1+x^{2}}, t \in J:=[0,5] \cap \mathbb{T}, 2<\alpha<3, \\
x(0)=x(5)=0, x^{\Delta}(0)=x^{\Delta}(5) .
\end{array}\right.
$$

Since $f(t, x)=\frac{x(1-\cos (t x))}{1+x^{2}} \in C([0,5] \times \mathbb{R}, \mathbb{R})$ satisfies $(H 5)$ and condition $(H 2)$ is satisfied, we see that BVP (4.1) has at least one solution by using Theorem 4.4.

\section{REFERENCES}

[1] A.S. Berdyshev, A. Cabada and B.Kh. Turmetov, On solvability of a boundary value problem for a nonhomogeneous biharmonic equation with a boundary operator of a fractional order, Acta Math. Sci. Ser. B Engl. Ed. 34 (2014), 16951706.

[2] F.T. Fen, I.Y. Karaca, O.B. Ozen, Positive solutions of boundary value problems for p-Laplacian fractional differential equations, Filomat, 31 (2017), 1265-1277.

[3] M. Günendi, İ. Yaslan, Positive solutions of higher-order nonlinear multi-point fractional equations with integral boundary conditions, Fract. Calc. Appl. Anal. 19 (2016), 989-1009.

[4] L. Kong, Q. Kong, M. Wang, Existence and uniqueness of solutions for a fractional boundary value problem with a separated boundary condition, Dyn. Syst. Appl. 23 (2014), 691-698.

[5] G.T. Wang, B. Ahmad, L. Zhang, Some existence results for impulsive nonlinear fractional differential equations with mixed boundary conditions, Comput. Math. Appl. 62 (2011), 1389-1397.

[6] G.T. Wang, B. Ahmad, L. Zhang, On nonlocal integral boundary value problems for impulsive nonlinear differential equations of fractional order, Fixed Point Theory, 15 (2014), 265-284.

[7] K.S. Miller, B. Ross, Fractional difference calculus, Proceedings of the International Symposium on Univalent Functions, Fractional Calculus and Their Applications, Nihon University, Koriyama, Japan 1989, 139-152. 
[8] F.M. Atici, P.W. Eloe, A transform method in discrete fractional calculus, Int. J. Differ. Equ. 2 (2007), 165-176.

[9] F.M. Atici, P.W. Eloe, Initial value problems in discrete fractional calculus, Proc. Amer. Math. Soc. 137 (2009), $981-989$.

[10] F.M. Atici, P.W. Eloe, Two-point boundary value problems for finite fractional difference equations, J. Difference Equ. Appl. 17 (2011), 445-456.

[11] R.A.C. Ferreira, Positive solutions for a class of boundary value problems with fractional q-differences, Comput. Math. Appl. 61 (2011), 367-373.

[12] C.S. Goodrich, Existence of positive solution to a system of discrete fractional boundary value problems, Appl. Math. Comput. 217 (2011), 4740-4753.

[13] C.S. Goodrich, On discrete sequential fractional boundary value problems, J. Math. Anal. Appl. 385 (2012), 111-124.

[14] T. Abdeljavad, D. Baleanu, Caputo q-fractional initial value problems and a q-analogue Mittag-Leffler function, Commun. Nonlinear Sci. Numer. Simul. 16 (2011), 4682-4688.

[15] P.A. Williams, Fractional calculus on time scales with Taylor's theorem, Frac. Calc. Appl. Anal. 15 (2007), $616-638$.

[16] G.A. Anastassiou, Foundations of nabla fractional calculus on time scales and inequalities, Comput. Math. Appl. 59 (2010), 3750-3762.

[17] F.M. Atici, P.W. Eloe, Fractional q-calculus on a time scale, J. Nonlinear Math. Phys. 14 (2007), 341-352.

[18] N.R.O. Bastos, D. Mozyrska, D.F.M. Torres, Fractional derivatives and integrals on time scales via the inverse generalized Laplace transform, Int. J. Math. Comput. 11 (2011), 1-9.

[19] G.A. Anastassiou, Principles of delta fractional calculus on time scales and inequalities, Math. Comput. Model. 52 (2010), 556-566.

[20] M. Bohner, A. Peterson, Advances in Dynamic Equations on Time Scales, Birkhäuser, Boston, 2003.

[21] M. Bohner, A. Peterson, Dynamic Equations on Time Scales: An Introduction with Applications, Birkhäuser, Boston, 2001.

[22] M. R. Sidi Ammi, D.F.M. Torres, Existence and uniqueness results for a fractional Riemann-Liouville nonlocal thermistor problem on arbitrary time scales, J. King Saud Univ. Sci. DOI: 10.1016/j.jksus.2017.03.004.

[23] J. Appell, B. Lopez, K. Sadarangani, Existence and uniqueness of solutions for a nonlinear fractional initial value problem involving Caputo derivatives, J. Nonlinear Var. Anal. 2 (2018), 25-33.

[24] İ. Yaslan, O. Liceli, Three-point boundary value problems with delta Riemann-Liouville fractional derivative on time scales, Frac. Differ. Calc. 6 (2016), 1-16.

[25] X. Zhang, C. Zhu, Cauchy problem for a class of fractional differential equations on time scales, Int. J. Comput. Math. 91 (2014), 527-538.

[26] M.A. Krasnoselskii, Positive Solutions of Operator Equations, Noordhoff, Groningen, 1964.

[27] J.X. Sun, Nonlinear Functional Analysis and Its Applications, Science Press, Beijing, 2008. 